\title{
Will Marley come home? An exploration of the impacts of the Canterbury earthquakes on people's relationships with their companion animals
}

\author{
Nikki Evans and Maria Perez-y-Perez
}

Nikki Evans and Maria Perez-y-Perez are both lecturers at the School of Human Services and Social Work, University of Canterbury.

\begin{abstract}
A sizeable number of New Zealand homes contain at least one companion animal - and many of these are afforded the status of family member by their human owner(s). It follows then that when a series of high-magnitude earthquakes shook the New Zealand city of Christchurch and the Canterbury region it is located within, many people and their companion animals were impacted. Generic and disaster-specific research into animal-human relationships has mostly been undertaken outside of the profession of social work. However, a number of recent social work research and theoretical papers draw attention to the need for this discipline to also embrace this field (Evans \& Gray, 2012; Morley \& Fook, 2005; Tedeschi, Fitchett, \& Molidor, 2005; Risley-Curtiss, Holley, \& Wolf, 2006b; Risley-Curtiss, 2010). The aftermath of the Canterbury earthquakes has revealed a need to look critically at how animal-human relationships are perceived, and the potential for these relationships to be considered within routine social work assessments and interventions. This paper considers the role of companion animals in people's lives, addresses the status of these animals during the Canterbury earthquakes, explores issues of loss and resiliency within animal-human relationships and looks at the implications of these relationships for social work practice and research.
\end{abstract}

\section{The status of companion animals before and during a natural disaster}

In Western culture at least, animals and children are considered to have something of a natural bond (Evans \& Gray, 2012; Heinsch, 2012; Melson \& Fine, 2006). This is evidenced in the vast array of toys, characters in books, television programmes, movies, computer games and imprints on clothing that are designed to represent countless animals such as bears, rabbits and dogs (Evans \& Gray, 2012).

Often begun in childhood, positive benefits of relationships formed between animals and people have been documented extensively within the human services literature (Evans \& Gray, 2012; Morley \& Fook, 2005). Many children in New Zealand grow up with a companion animal, and this country has recently been identified as having a higher rate of companion animal ownership than any comparable country in the Western world. In fact, $68 \%$ of 
New Zealand households are reported to include at least one companion animal (MacKay, 2011). It is easy to extrapolate from this statistic that the seismic events in the Canterbury region that began on the 4th September 2010 will have impacted on many households with companion animals, as well as on the animals themselves. In all likelihood, when each major earthquake occurred in this region, large numbers of houses and some commercial buildings would have contained not only people but the companion animals that they were responsible for (Evans, 2011).

There is no doubt that these companion animals have played a significant role in the actions and lives of many people in the Canterbury region - and many of these animals will have been given the status of family member by their human owners (Walsh, 2009). This aspect of companion animal ownership is such an intrinsic part of New Zealand culture that the Society for the Prevention of Cruelty to Animals (SPCA) has used it as leverage in their 'One of the Family' campaign - a campaign that is designed to raise awareness about the link between animal cruelty and violence toward people (Glassey, 2010).

The SPCA was also involved in rescue and recovery activities during the Canterbury earthquakes. Following the magnitude 6.3 earthquake in Christchurch city on February 22, 2011, the Wellington branch's Animal Rescue Unit (ARU) played a significant role in the rescue and recovery of many animals. The SPCA's National President, Bob Kerridge, said that 'Whilst the emergency services concentrated on saving human lives, the SPCA took the primary responsibility for caring for animal survivors and, wherever possible, reuniting lost pets with their owners' (Kerridge, 2011). Members of Massey University's Veterinary Emergency Response Team, the first of its kind in Australasia, were also deployed to join SPCA employees and volunteers. However, with the exception of these national and local specialist services, and some lay people who focused their attention on rescue and recovery of animals, most prioritised rescue and recovery of people (Evans, 2011). There is clearly some question about whether companion animals retain the status of 'family members' during times of crisis.

New Zealand is definitely not alone in its need to critically explore the status of animals within society (Evans, 2011). Recent disasters in the United States of America have drawn attention to seemingly contradictory approaches to animal-human relationships and the relatively low status afforded animals in times of crisis or disaster (Evans, 2011). When Hurricane Katrina destroyed large parts of New Orleans, rescuers were either not allowed to assist companion animals of the people they rescued - or they chose not to (Barton Ross \& Baron-Sorensen, 2007). Likewise, after the 9/11 terrorist attacks in New York companion animals were left to fend for themselves, with many perishing in apartments that were abandoned or cordoned (Evans, 2011). Similar criticisms about animal welfare management emerged in Australia following the bushfires in Victoria in 2009 and the floods in Queensland in 2010-11 (White, 2012).

Ensuing public outcry about animal welfare in disaster contexts has led to a questioning of the status of companion animals in disaster recovery efforts. Many authors in the disaster management field have argued that subsequent policy developments in several countries still inadequately address the needs of companion animals (Akhtar, 2012; Barton Ross \& Baron-Sorensen, 2007; Evans, 2011; Glassey \& Wilson, 2011; White, 2012). 
Evacuation plans that neglect the retrieval and care of companion animals can compromise the wellbeing of both the animals and their owners. Yet during Hurricane Katrina, emergency shelters did not allow pets (Akhtar, 2012). While the rescue and recovery efforts and scale of evacuations were comparatively small, anecdotal evidence does suggest that Canterbury residents experienced issues in relation to their companion animals following each of the significant earthquakes.

Public expectations of authorities in relation to companion animals can be out of sync with legislation and local policy, leading to a range of significant issues for authorities in disaster situations (Evans, 2011; White, 2012). It has become clear that individual animal owner community, and national disaster response plans do not always include the rescue and care of companion animals. However, in reality, the deep connection some people feel with companion animals is likely to lead some people to ignore directives of officials if they feel that they will compromise their animal's welfare. This approach relies on people having capacity, knowledge and resources to adequately address the needs of companion animals in a disaster context - something that may well be contestable (White, 2012).

People can and do refuse to evacuate buildings or areas without their companion animals. Some choose instead to remain in dangerous situations themselves, accepting that they may lose their own life trying to protect their animals (Akhtar, 2012; Barton Ross \& Baron-Sorensen, 2007; Glassey \& Wilson, 2011). Failure of emergency response efforts to take care of companion animals clearly compromises human lives (Glassey, 2010).

Official disaster response plans must be realistic about the fact that a large number of people in New Zealand have companion animals or animals that they feel responsible for. Having members of the public ignore official processes and policy in disaster situations is clearly unacceptable for a range of reasons. Anecdotal reports suggest that following the February 22 earthquake in Christchurch, some people broke official cordons to save animals, put themselves in danger by refusing to leave condemned homes or buildings until their companion animal was found, were unable to transport their animals to new accommodation, take animals into shelters set up for people, or find temporary accommodation that allowed them to have pets. Some authors have noted that at the time of this significant earthquake, there was no statutory requirement for animal welfare to be considered by Civil Defence Emergency Management Groups in New Zealand (Glassey \& Wilson, 2011).

Fortunately, while the Canterbury earthquakes had many devastating impacts, what needed to be dealt with was on a much smaller scale than either Hurricane Katrina or the 9/11 terrorist attacks (Evans, 2011). However, many people in the region did not have a personal disaster plan or adequate supplies for themselves or their companion animals. And, interestingly, public campaigns in New Zealand about disaster preparedness did not feature companion animals and barely addressed the issue of responsibility for animals in a disaster (Evans, 2011).

There is a need for individual animal owners to develop a personal plan for the evacuation and care of their animals - but this must be matched by official responses. Evacuation plans need to be developed at local and national levels that are based on 'the assumption that evacuated companion animals will need to be accommodated' (Glassey, 2010, p.7). There is a clear need for national and international emergency policies and protocols to include 
appropriate management of companion animals for the physical and psychological welfare of both the animals and their owners (Akhtar, 2012; Barton Ross \& Baron-Sorensen, 2007; Glassey \& Wilson, 2011; Irvine, 2009).

\section{Dealing with lost and found animals}

Following the immediate impacts of a natural disaster, people are often faced with other problems, including housing issues and the loss of animals. Incredibly, Hurricane Katrina is estimated to have affected 727,500 pets in the city of New Orleans alone (Irvine, 2009). After Hurricane Katrina, and the 2011 earthquake and tsunami in Japan, many stories have been reported in the media about people being reunited with lost animals. After the 2010/2011 Canterbury earthquakes, many stories told to us informally, similar to the one below, emerged:

Alison searched for days on foot for her beloved cat Larry, walking along broken pavements, twisted bridges, and through liquefaction as she searched. Her husband drove around local streets in their four-wheel drive until he eventually spotted Larry on top of a fence several blocks away. The fence that Larry was sitting on was surrounded by water and liquefaction and he had been too afraid to jump down and find his way home. When he was found, Larry had been missing for three days.

Following the crisis period after the February 22, 2011, magnitude 6.3 earthquake many stranded animals were lost - and others, like Larry, were found. Local animal welfare services were inundated with animals and requests for assistance with animals (Evans, 2011). The Canterbury branch of the SPCA dealt with many hundreds of animals during this period of time and had a range of systems in place to track and locate lost and found pets (Glassey \& Wilson, 2011). Large numbers of animals in the Canterbury region, especially those that had been microchipped, were able to be reunited with their owners. Another key measure was the use of web-based systems that listed lost and found animals, and there were anecdotal reports of people around New Zealand and even some people in Australia spending time perusing online posts to facilitate the matching of lost pets with their owners.

Not all animals were found or able to be returned to their owner(s). The loss of a companion animal in any circumstance can have a significant impact upon people (Barton Ross \& Baron-Sorensen, 2007; Blazina, Boyraz, \& Shen-Miller, 2011; Morley \& Fook, 2005; Myers, 2002). When the loss of a companion animal occurs within the context of a natural disaster, with its own associated trauma, a person's recovery and resilience may be compromised especially for those people for whom the companion animal is a part of their usual support system (Evans \& Gray, 2012). An example of this kind of loss can be seen in the following vignette, based on a story told to us:

Paddy never found his cat Blackie after the first earthquake in September 2010. He talked about the many times when he felt stressed, initially in the immediate aftermath of the earthquakes when he felt scared, later when he had to move out of his badly damaged home, and later still when he struggled in his dealings with the Earthquake Commission (EQC - a New Zealand Government agency that provides natural disaster insurance to residential property owners). Paddy talked about how he longed, during these times, to sit on his sofa and stroke Blackie an activity that he had always found grounding and soothing. 
The significance of the loss of a companion animal will differ between people and can be influenced by many things including:

- the role of the animal in the emotional and physical health of the person,

- length of time the person had the animal in their life,

- significant life events experienced while the animal had been in their life (for instance, divorce, cancer treatment),

- the significance of the animal in their life journey (for instance, a belief that the animal saved their life),

- the circumstances surrounding the loss,

- changes to social networks and daily routines (i.e., not going to the dog park each night),

- the financial or intrinsic value of the animal,

- the role of the animal in a breeding programme, and

- the social reactions to the loss.

(Evans, 2011, p.23).

The meanings people make about companion animals in their lives, and the ways in which they reveal the impact of the death or loss of an animal, are largely defined by the social construction of the animal-human bond (Morley \& Fook, 2005). This bond is generally interpreted in comparison with human companionship, revealing an implicit assumption that human-human relationships are the normative measure - they are the ideal (Morley \& Fook, 2005). It follows that if a person is perceived as being 'too close' to an animal, they are in some way lacking or deficient in their capacity to have fulfilling relationships with other humans. Therefore, the deep connection some people experience with companion animals, and the experience of loss of that animal when it dies, is often minimised or pathologised (Morley \& Fook, 2005).

When the bond between people and animals is seen as a substitute for human contact there is a flow on effect about how people 'ought' to react when an animal is lost or dies (Morley \& Fook, 2005). The notion that companion animals continue to hold the status of esteemed family members in times of loss is clearly highly contestable. Such assumptions about the status of companion animals in people's lives can prevent people talking about the loss experienced when the animal has died or disappeared (Evans, 2011). Family, friends, colleagues, employers, doctors, social workers and even veterinary practitioners can belittle the loss of a companion animal, compounding the person's sense of isolation, sorrow and grief (Myers, 2002).

People can feel embarrassed about the intensity of their feelings and can go to extraordinary lengths to conceal their feelings of loss in relation to a companion animal from those around them (Barton Ross \& Baron-Sorensen, 2007; Morley \& Fook, 2005; Myers, 2002). In the context of disaster that has claimed human and animal lives, people may be even more reluctant to disclose their experiences of companion animal loss - something that critical response teams, social workers and other human service practitioners need to be alert to.

\section{Marking the end of the animal-human relationship}

Life transitions or endings are often marked in ritualised ways. We only have to look as far as milestone birthdays, weddings or civil union ceremonies, graduations and funerals to find examples (Evans, 2011). Yet within Western cultures, there is little clarity, social acknowledgement and ritual around the death of an animal (Evans, 2011; Myers, 2002). 
Whatever family or community traditions have developed over time, marking the end of a particular animal-human relationship and the process of farewelling a companion animal will be altered if it occurs within the context of a disaster where people may need to make decisions quickly and their choices may be restricted (Evans, 2011).

Acceptance of people's need to talk about and mark the lives of companion animals with some form of ending process, by social workers and others working in the immediate aftermath of a disaster, may be quite critical to their client's recovery from the loss.

\section{Forced loss: Abandonment and relinquishment}

Saying goodbye to a companion animal is not always about the death of the animal. Sometimes it is about clients who have needed to leave behind or relinquish companion animals. The decision to leave an animal behind without being sure of its safety or wellbeing can result in a form of survivor guilt or significant grief responses, and is, of course, illegal in New Zealand (Evans, 2011). Owners of companion animals retain statutory responsibility for the care of their animals - even during a disaster. So aside from the emotional responses a person may have to abandoning a companion animal, failure to provide for companion animals is a criminal offence in New Zealand (Glassey, 2010).

Yet it may be physically impossible, in the midst of a disaster, for people to transport the companion animals in private motor vehicles or unrestrained on public transport that does not allow pets. There is some contention about the notion of owner responsibility for the care of animals in situations where people have been evacuated or forcibly removed from their property. In these situations an argument for statutory responsibility seems plausible (Glassey, 2010).

The sense of emotional, moral and legal responsibility for animals was noted to be a significant issue for people after Hurricane Katrina, but was also an issue in Christchurch and one that compelled people to disregard official cordons in an endeavour to save animals that they were responsible for (Evans, 2011).

Relinquishment of a companion animal is another difficult process for people - and one that they may seek professional assistance with. In the context of a disaster, relinquishment can happen for a range of reasons: shelters may be full; temporary accommodation may not allow pets; owners may be staying with extended family where there are already many pets; financial issues; changes in the animal's behaviour that are difficult to manage; and convenience (Evans, 2011). It is important that social workers intervening with people in disaster contexts are aware that the relinquishment of a companion animal can mean a loss of a significant support for the individual or family (Faver \& Cavazos, 2008; Kurdek, 2009; Walsh, 2009), and compound the earthquake-related distress experienced by the owner(s) (Barton Ross \& Baron-Sorensen, 2007).

Relinquishment of a loved companion animal also involves a process of saying goodbye and this can be an especially difficult experience as the following vignette, about a man who 're-homed' his much loved golden retriever dogs illustrates:

[He] had a farewell bacon sandwich with his two old friends ... 'It was very sad. We had a last bacon sandwich together - me, George and Mildred in the lounge. That was the last meal 
we had together,' he said ... 'I gave them their bowls and leads and just said goodbye, which really hurt. I didn't look when they were driven away. I just went in the house' (Gates, 2011, cited in Evans, 2011).

Animal-human relationships must be taken seriously in both response and recovery plans for disasters within the New Zealand context. Acknowledgement of the impact of changes to the animal-human relationship or loss of a companion animal also need to feature in assessments by critical response teams, social workers and other human service practitioners. If these workers ensure the wellbeing and safety of humans in the fullest sense, the wellbeing and safety of companion animals will also have been attended to (Akhtar, 2012; Glassey \& Wilson, 2011).

\section{Altered relationships: The impact of earthquakes on the animal-human bond}

The support, stability, comfort and unconditional love a companion animal can provide to people in a time of crisis should not be underestimated (Kurdek, 2009). The support that people receive from their relationship with a companion animal can be an adjunct to or in place of support from other people. It can occur when support from other people has not been sought, offered or taken up (Barton Ross \& Baron-Sorensen, 2007; Evans, 2011; Evans \& Gray, 2012; Kurdek, 2009; Melson \& Fine, 2006). Indeed, social support is known to reduce stress in people and is related to resilience in the face of adversity - including natural disasters (Evans, 2011; Evans \& Gray, 2012; Melson \& Fine, 2006). In many disaster situations, the support that people have sought and that animals have given during difficult times may increase.

Given that animals can provide strong emotional and psychological support for people, and are frequently regarded as members of the family, it is important to consider what happens when this support is removed - not only when the animal has been lost, but also when the nature of the relationship has been altered by a natural disaster. Changes to the dynamics of the animal-human relationship after a disaster are largely unexplored within research in this field, and is likely to be similarly unexplored by practitioners working with clients within a disaster context.

Social workers need to be aware of possible issues that may impact on, and at times strain, the animal-human relationship including: changes in the animal's behaviour, such as the development of anxious or aggressive behaviour; refusal of animals to return to the house after a major earthquake; living in temporary accommodation that is overcrowded or not suited to the animal's needs; living with an 'inside' animal that has to adapt to becoming an 'outside' animal or vice versa (Evans, 2011). An example of such a change can be seen in the following vignette, based on another story that was told to us informally:

Mary lived rurally, and her dogs were her constant companions. She reported that her dogs were extremely well socialised and well trained. However, immediately following the September 4, magnitude 7.1 earthquake in Canterbury, one of Mary's dogs became very anxious and extremely aggressive to her other dog. The problem escalated with continuing aftershocks to the point where Mary could not leave the dogs unsupervised. What was a relaxing and supportive relationship between owner and dogs became stressful and unsustainable. 
A further example told to the authors relates to a cat that would not return to the house following a large earthquake.

Gina described her experience of her cat tearing out of the house, where it had been curled up sleeping, when the September 4 earthquake happened. She reflected that it took 18 months for the cat to enter the house again, and saw this act as a significant step in her entire family's recovery.

It is important for social workers to acknowledge changes in the animal's behaviour or to the nature of the animal-human relationship as a form of loss (Evans, 2011). These kinds of stressors may not be readily visible to social workers and other human service practitioners - unless they ask about them.

\section{Environmental challenges and creating social capital}

Social work practice within the Canterbury context will continue to be challenging as workers and their clients continue to negotiate unchartered territory. Perhaps more than ever before, the notion of person-in-the-environment is relevant to social work assessment and intervention in this region. Relationships with companion animals can also be understood in terms of the interaction between the person and their environment, and occur within the constraints of many physical contexts, including homes, residential streets and neighbourhoods, parks and beaches.

Relationships, trust, connections, social norms, and connections between people, animals and 'things' can interact to produce economic and collective benefits that in turn lead to healthy communities or social capital. Interestingly, companion animals can contribute significantly to the development of social capital. They can be facilitators, getting people out using community facilities and engaged in community events (Wood, Giles-Corti, Bulsara, \& Bosch, 2007). Indeed, research has shown that more animal owners than non-owners engaged in neighbourly acts of reciprocity (for instance, feeding animals, lending items, exchanging favours) - a hallmark of social capital (Wood, et al., 2007; Wood, 2012).

Companion animal ownership can create habits of a lifetime and for many Christchurch dog owners, a walk around the block, along the beach, in a dog park or to a local reserve would have been a common activity. Such activities are not only about exercise for the dog - they can also provide a platform for key human benefits including exercise, lifestyle balance, contact with others in the neighbourhood and sometimes perceptions of increased community safety (Wood, et al., 2007).

Interesting stories about people checking on their neighbours following large earthquakes have emerged over time. For some of these people, they only knew their neighbour as the owner of 'Freddy' or 'Rosy' - the dog that lived down the road. One such story was told to us.

Once he had made sure his human family and companion animals were safe, Barry went down the road to check on elderly neighbours he had met over the years when out walking his dogs. A connection established through the dogs motivated him to move quickly to check on these neighbours after significant earthquakes struck the region. 
Within the changed physical and social environments created by the Canterbury earthquakes, people continue to negotiate their relationships with each other and their companion animals. When the physical environment is altered by events such as earthquakes, and familiar walking routes, parks and facilities becoming inaccessible or contaminated, people can experience further stress-evoking change. The impact of the loss of social contact, exercise opportunity and routine should not be underestimated by social workers - nor should the impact of having a dog in the (possibly temporary) house, which has not been walked for several days! (Evans, 2011).

As the immediate sequelae of the earthquakes passed, many people turned their attention to the re-build of the city of Christchurch. Anecdotal reports suggest that for some people the re-build has thrown up new challenges and concerns. Some people have expressed concern about an apparent lack of animal-friendly spaces, or areas conducive to fostering animal-human activities within emerging city plans. There is a growing interest in the positive ripple effects that companion animals and animal ownership can have on the broader community (Wood et al., 2007).

Some authors have noted that animal-related activities often times bring people closer to 'nature'. An association with nature, in the form of backyards, parks, trees, beaches and communal spaces can also have a positive impact on both animal and human wellbeing (Heinsch, 2012) and for this reason ought to be a focus of re-building a city or region. A focus on re-building community capacity and social capital is likely to persist for some time in Christchurch and social workers will have opportunities to proactively support initiatives that get people (and their companion animals) together.

\section{Social work education and practice}

Somewhat fortuitously, social work education and practice have embraced ecological frameworks for many years. Social workers have long accepted the premise that people develop and adapt through dynamic and reciprocal transactions with all components of their environment (Tedeschi, et al., 2005; Zastrow, 2008) and this is being evidenced on a daily basis in Canterbury. However, despite the person-in-the-environment approach that characterises the profession, a focus on human-animal relationships has been largely absent. The ripple effects that these relationships and animal ownership per se, can have on the functioning of the broader community have received minimal attention from social work educators, researchers and practitioners to date (Evans \& Gray, 2012; Risley-Curtiss, et al., 2006b).

Many social workers have occasion to interact with companion animals when on home visits to clients and the opportunity to acknowledge these animals as members of the family and this is probably never more important than in times of disaster or significant crisis (Boat and Knight, 2000; Evans \& Gray, 2012; Kurdek, 2009; Tedeschi et al., 2005; Risley-Curtiss et al., 2006a; Risley-Curtiss, 2010; Walsh, 2009). With a greater awareness of the significance and implications of animal-human relationships within the post-earthquake context, social workers in a range of fields can and should develop assessments and interventions accordingly (Risley-Curtiss et al., 2006a; Risley-Curtiss, 2010).

Perhaps even more so than usual, social workers practising in a time of natural disaster may need to manage difficult situations, such as encounters with aggressive animals, or 
conflicts about animal 'custody', relinquishment or welfare (Boat \& Knight, 2000), as well as situations of hoarding that may have been exposed by visits by civil defence or EQC personnel. For some clients, leaving animals at home while they attend appointments is seen as untenable. Fear that an earthquake will occur while they were away may lead some clients to choose to miss appointments or ask to bring companion animals with them to meetings. Social workers practising in these contexts need to be clear about their own expectations and boundaries as well as be flexible and responsive to the changed contexts of clients' lives.

In reality, social workers may be poorly equipped to deal with animal-human relationships within the bounds of their professional practice as there is negligible focus on these relationships within social work education and research (Evans \& Gray, 2012; Risley-Curtiss et al., 2006b).

\section{Where to from here?}

Whether we accept the challenge to consider animal-human relationships within the core social work education curriculum (Evans \& Gray, 2012; Risley-Curtiss, et al., 2006b; Wolf, 2000) or not, the need for social workers to include a focus on animal-human relationships in assessment and sometimes intervention within the context of a disaster is beyond refute. Yet there is very little research that can support social work practitioners intervening with people who have lost or relinquished companion animals in the context of an earthquake, or had their relationship altered by the ongoing seismic activity. The more we recognise animal-human bonds through research and theory development then the more social workers and other human service practitioners can legitimise clients' diverse reactions to loss or change to these relationships during a natural disaster.

The study of animal-human relationships is a complex and at times contentious area of enquiry. However, it is not enough to leave research and practice development in this area to a small group of sympathetic researchers and practitioners - it is time for the discipline to ensure that the focus on the person-in-the-environment includes a focus on the person and their animal(s).

\section{References}

Akhtar, D. A. (2012). Animals and public health: Why treating animals better is critical to human welfare. Basingstoke, England: Palgrave Macmillan.

Barker, S. B., \& Wolen, A. R. (2008). The benefits of human-companion animal interaction: A review. Journal of Veterinary Medical Education, 35, 487-495.

Barton Ross, C., \& Baron-Sorensen, J. (2007). Pet loss and human emotion: A guide to recovery. New York, NY: Routledge.

Beck, A. M., \& Katcher, A. H. (2003). Future directions in human-animal bond research. American Behavioral Scientist, 47, 79-93.

Blazina, C., Boyraz, G., \& Shen-Miller, D. (Eds.). (2011). Using context to inform clinical practice and research. The psychology of the human-animal bond: A resource for clinicians and researchers (pp.2-24). New York, NY: Springer.

Boat, B. W., \& Knight, J. C. (2000). Experiences and needs of adult protective services case managers when assisting clients who have companion animals. Journal of Elder Abuse E Neglect, 12, 145-155.

Evans, N. (2011). The dynamics of animal-human relationships during and following a natural disaster. Te Awatea Review, 9(1\&2), 22-25.

Evans, N., \& Gray, C. (2012). The practice and ethics of animal-assisted therapy with children and young people: Is it enough that we don't eat our co-workers? British Journal of Social Work, 42, 600-617.

Faver, C. A., \& Cavazos, A. M. (2008). Love, safety, and companionship: The human-animal bond and Latino families. Journal of Family Social Work, 11, 254-271.

Gates, C. (2011, June 23). Dogs bring post-quake cheer. The Press. Retrieved from http: / / www.stuff.co.nz / the-press / news / christchurch-earthquake-2011/5180385/Dogs-bring-post-quake-cheer. 
Glassey, S. (2010). Recommendations to enhance companion animal emergency management in New Zealand. Wellington: Mercalli Disaster Management Consulting.

Glassey, S., \& Wilson, T. (2011). Animal welfare impact following the 4 September 2010 Canterbury (Darfield) earthquake. Australasian Journal of Disaster and Trauma Studies, 2, 49-59.

Hart, L. (Ed.). (2000). Psychological benefits of animal companionship. San Diego, CA: Academic Press.

Heinsch, M. (2012). Getting down to earth: Finding a place for nature in social work practice. International Journal of Social Welfare, 21, 309-318.

Irvine, L. (2009). Filling the ark: Animal welfare in disasters. Philadelphia, PA: Temple University Press.

Kerridge, B. (2011). SPCA hails its Christchurch heroes. Press release retrieved from http://rnzspca.org.nz/about/ news / press-releases / 377-spca-hails-its-christchurch-heroes.

Kurdek, L. A. (2009). Pet dogs as attachment figures for adult owners. Journal of Family Psychology, 23, 439-446.

MacKay, J. (2011). Companion animals in New Zealand. New Zealand Companion Animal Council Inc.

Melson, G. F., \& Fine, A. H. (2006). Animals in the lives of children. In A. H. Fine (Ed.), Handbook on animal-assisted therapy: Theoretical foundations and guidelines for practice (2nd ed., pp. 207-226). San Diego, CA. Academic Press.

Morley, C., \& Fook, J. (2005). The importance of pet loss and some implications for services. Mortality, 10(2), $127-143$.

Myers, B. (2002). Disenfranchised grief and the loss of an animal companion. In K. J. Doka (Ed.), Disenfranchised grief: New directions, challenges, and strategies for practice (pp.251-264). Champaign, IL: Research Press.

Risley-Curtiss, C. (2010). Social work practitioners and the human-animal bond: A national study. Social Work, 55(1), 38-46.

Risley-Curtiss, C., Holley, L. C., Cruickshank, T., Porcelli, J., Rhoads, C., Bacchus, D. N. A., Nyakoe, S., \& Murphy, S. B. (2006a). She was family: Women of color and animal-human connections. Affilia, 21(4), $433-447$.

Risley-Curtiss, C., Holley, L. C., \& Wolf, S. (2006b). The animal-human bond and ethnic diversity. Social Work, 51(3), 257-268.

Tedeschi, P., Fitchett, J., \& Molidor, C. E. (2005). The incorporation of animal-assisted interventions in social work education. Journal of Family Social Work, 9(4), 59-77.

Walsh, F. (2009). Human-animal bonds II: The role of pets in family systems and family therapy. Family Process, $48,481-499$.

Wells, D. L. (2009). The effects of animals on human health and well-being. Journal of Social Issues, 65, 523-543.

White, S. (2012). Companion animals, natural disasters and the law: An Australian perspective. Animals, 2, $380-394$.

Wolf, D. B. (2000). Social work and speciesism. Social Work, 45, 88-93.

Wood, L. J., Giles-Corti, B., Bulsara, M. K., \& Bosch, D. A. (2007). More than a furry companion: The ripple effect of companion animals on neighborhood interactions and sense of community. Society and Animals, 15(1), 43-56.

Wood, L. (2012). The pet connection. Paper presented at the 23rd NZ Companion Animal Conference, 10th October 2012. Wellington, New Zealand.

Zastrow, C. (2008). Introduction to social work and social welfare: Empowering people (9th ed), Belmont, CA, Thompson Brooks / Cole. 